\title{
Observation of GRB 030131 with the INTEGRAL satellite ${ }^{\star}$
}

\author{
D. Götz ${ }^{1,2}$, S. Mereghetti ${ }^{1}$, K. Hurley ${ }^{3}$, S. Deluit ${ }^{4}$, M. Feroci ${ }^{5}$, F. Frontera ${ }^{6,7}$, A. Fruchter ${ }^{8}$, J. Gorosabel $^{8,16}$, \\ D. H. Hartmann ${ }^{9}$, J. Hjorth ${ }^{10}$, R. Hudec ${ }^{11}$, I. F. Mirabel ${ }^{12,15}$, E. Pian ${ }^{13}$, G. Pizzichini ${ }^{7}$, P. Ubertini ${ }^{5}$, and C. Winkler ${ }^{14}$ \\ ${ }^{1}$ Istituto di Astrofisica Spaziale e Fisica Cosmica - CNR, Sezione di Milano “G. Occhialini”, Via Bassini 15, 20133 Milano, \\ Italy \\ 2 Dipartimento di Fisica, Università degli Studi di Milano Bicocca, P.zza della Scienza 3, 20126 Milano, Italy \\ 3 UC Berkeley Space Sciences Laboratory, Berkeley CA 94720-7450, USA \\ 4 Integral Science Data Centre, Chemin d'Écogia 16, 1290 Versoix, Switzerland \\ 5 Istituto di Astrofisica Spaziale e Fisica Cosmica - CNR, via Fosso del Cavaliere 100, 00133 Roma, Italy \\ ${ }^{6}$ Dipartimento di Fisica, Università di Ferrara, Via Paradiso 12, 44100 Ferrara, Italy \\ 7 Istituto di Astrofisica Spaziale e Fisica Cosmica - CNR, Sezione di Bologna, via Piero Gobetti 101, 40129 Bologna, Italy \\ ${ }^{8}$ Space Telescope Science Institute, 3700 San Martin Drive, Baltimore, MD 21218, USA \\ 9 Department of Physics and Astronomy, Clemson University, Clemson, SC 29634-0978, USA \\ 10 Astronomical Observatory, University of Copenhagen, Juliane Maries Vej 30, 2100 Copenhagen, Denmark \\ 11 Astronomical Institute, Academy of Sciences of the Czech Republic, 25165 Ondrejov, Czech Republic \\ 12 Service d'Astrophysique, CEA/Saclay, Orme des Merisiers, Bât. 709, 91191 Gif-sur-Yvette, France \\ 13 Osservatorio Astronomico di Trieste, Via GB Tiepolo 11, 34131 Trieste, Italy \\ 14 ESA-ESTEC, RSSD, Keplerlaan 1, 2201 AZ Nordwijk, The Netherlands \\ 15 Instituto de Astronomia y Fisica del Espacio / CONICET, cc67, suc 28. 1428 Buenos Aires, Argentina \\ 16 Instituto de Astrofísica de Andalucía (IAA-CSIC), PO Box 03004, 18080 Granada, Spain
}

Received 3 June 2003 / Accepted 23 July 2003

\begin{abstract}
A long Gamma-Ray Burst (GRB) was detected with the instruments on board the INTEGRAL satellite on January 31 2003. Although most of the GRB, which lasted $\sim 150 \mathrm{~s}$, occurred during a satellite slew, the automatic software of the INTEGRAL Burst Alert System was able to detect it in near-real time. Here we report the results obtained with the IBIS instrument, which detected GRB 030131 in the $15 \mathrm{keV-200} \mathrm{keV} \mathrm{energy} \mathrm{range,} \mathrm{and} \mathrm{ESO/VLT} \mathrm{observations} \mathrm{of} \mathrm{its} \mathrm{optical} \mathrm{transient.}$ The burst displays a complex time profile with numerous peaks. The peak spectrum can be described by a single power law with photon index $\Gamma \simeq 1.7$ and has a flux of $\sim 2$ photons $\mathrm{cm}^{-2} \mathrm{~s}^{-1}$ in the $20-200 \mathrm{keV}$ energy band. The high sensitivity of IBIS has made it possible for the first time to perform detailed time-resolved spectroscopy of a GRB with a fluence of $7 \times 10^{-6} \mathrm{erg} \mathrm{cm}^{-2}$ (20-200 keV).
\end{abstract}

Key words. gamma rays: bursts - gamma rays: observations

\section{Introduction}

Ever since their discovery (Klebesadel et al. 1973), Gamma-Ray Bursts (GRBs) have been a puzzling mystery, mostly because of their short durations and the apparent lack of counterparts at other wavelengths. A breakthrough in this field came thanks to the Italian-Dutch satellite BeppoSAX, which had the capability to localize the bursts' prompt emission with a precision of a few arcminutes within a few

Send offprint requests to: $\mathrm{D}$. Götz,

e-mail: diego@mi.iasf.cnr.it

* Based on observations with INTEGRAL, an ESA project with instruments and science data centre funded by ESA member states (especially the PI countries: Denmark, France, Germany, Italy, Switzerland, Spain), Czech Republic and Poland, and with the participation of Russia and the USA, and on observations collected by the Gamma-Ray Burst Collaboration at ESO (GRACE) at the European Sourthern Observatory, Paranal, Chile (Programme 70.D-0523). hours. This led to the discovery of the afterglow emission at lower energies, initially in X-rays (Costa et al. 1997) and subsequently at optical (van Paradijs et al. 1997) and radio (Frail et al. 1997) wavelengths, which allowed the redshift of these objects to be measured, and firmly established the cosmological nature of GRBs.

Due to the limited duration and the fading character of the afterglow emission, the prompt distribution of GRB coordinates to the scientific community is a high priority. After the end of the BeppoSAX mission this task has been accomplished mainly by HETE-2 (Ricker et al. 2002). INTEGRAL (Winkler et al. 1999), although not specifically designed as a GRB-oriented mission, can contribute to the rapid localization of the prompt emission of GRBs thanks to the INTEGRAL Burst Alert System (IBAS; Mereghetti et al. 2001). This software, running at the INTEGRAL Science Data Centre (ISDC; Courvoisier et al. 1999), is able to detect and localize GRBs 
with a precision of a few arcminutes in a few seconds, and to distribute their coordinates in near real time over the Internet.

The high sensitivity of the INTEGRAL instruments also allows us to study in detail the prompt $\gamma$-ray emission of GRBs. This is particularly interesting for the faintest bursts, for which deep spectral studies were not possible up to now. For example, with the CGRO/BATSE instrument, time resolved spectroscopy was possible only for bursts with a fluence larger than $\sim 4 \times 10^{-5}$ ergs cm$^{-2}$ (Preece et al. 1998).

On January 312003 at 07:38:49 UTC a GRB was detected in the field of view of the main instruments on board INTEGRAL: IBIS (Ubertini et al. 1999) and SPI (Vedrenne et al. 1999). Here we concentrate on the results obtained with IBIS, a coded mask imaging telescope based on two detectors, ISGRI and PICsIT, operating in the $15 \mathrm{keV}-1 \mathrm{MeV}$ and $170 \mathrm{keV}-10 \mathrm{MeV}$ energy ranges, respectively.

\section{Detection and localization}

GRB 030131 was discovered by IBAS (using IBIS/ISGRI data) on January 312003 at 07:39:10 UTC ( 21 s after the beginning of the GRB, see below). The on-line automatic imaging analysis localized it to off-axis angles $Z=10.1^{\circ}, Y=-3.6^{\circ}$, in the partially coded field of view (only $\sim 23 \%$ of the detector was illuminated by the GRB). The GRB coordinates were not distributed automatically by IBAS because most of the burst occurred during a satellite slew (IBAS is disabled during satellite slews). In fact only the first $\sim 20$ s of this $\sim 150$ s long burst, during which the satellite attitude was stable and well known, were analyzed by IBAS, resulting in a low significance of the trigger. An off-line interactive analysis confirmed the reality of the event (Borkowski et al. 2003), but the reported error region radius was underestimated. A correct localization with an error radius of 5' (Mereghetti et al. 2003a) was distributed only three days later.

By accumulating data over short time intervals and analyzing the corresponding images, we confirmed that the satellite slew started at 07:39:09 UTC, as indicated by the attitude data. We therefore used the first $20 \mathrm{~s}$ of the event, corresponding to the stable pointing period, to derive the GRB position $\alpha_{\mathrm{J} 2000}=13^{\mathrm{h}} 28^{\mathrm{m}} 21^{\mathrm{s}}, \delta_{\mathrm{J} 2000}=+30^{\circ} 40^{\prime} 33^{\prime \prime}$, with an error radius of $2.5^{\prime}$. Although the statistical error in these coordinates is only $1.6^{\prime}$, we conservatively added a systematic uncertainty of $2^{\prime}$, based on the results obtained in IBIS observations of sources with known positions. Our final position for GRB 030131 is consistent with the one reported earlier (Mereghetti et al. 2003a) and with the annulus derived with the IPN using Ulysses and IBIS/ISGRI data (see Fig. 1).

A provisional identification of an optical transient (OT) for GRB 030131 was reported by Fox et al. (2003a). The candidate OT, detected with the Palomar 48-inch Oschin telescope + NEAT Camera, had magnitude $R \sim 21.2$ at 3.62 hours after the burst, but it was much fainter and barely detectable $(R>23.5)$ $\sim 26.8$ hours after the burst at the 200-inch Hale telescope. Its coordinates, $\alpha_{\mathrm{J} 2000}=13^{\mathrm{h}} 28^{\mathrm{m}} 22.29^{\mathrm{s}}, \delta_{\mathrm{J} 2000}=+30^{\circ} 40^{\prime} 23.7^{\prime \prime}$, are $20^{\prime \prime}$ from the center of the error circle derived here.

As a follow-up, we obtained a $3 \times 300 \mathrm{~s}$ exposure in the $V$ band using the European Southern Observatory Very Large

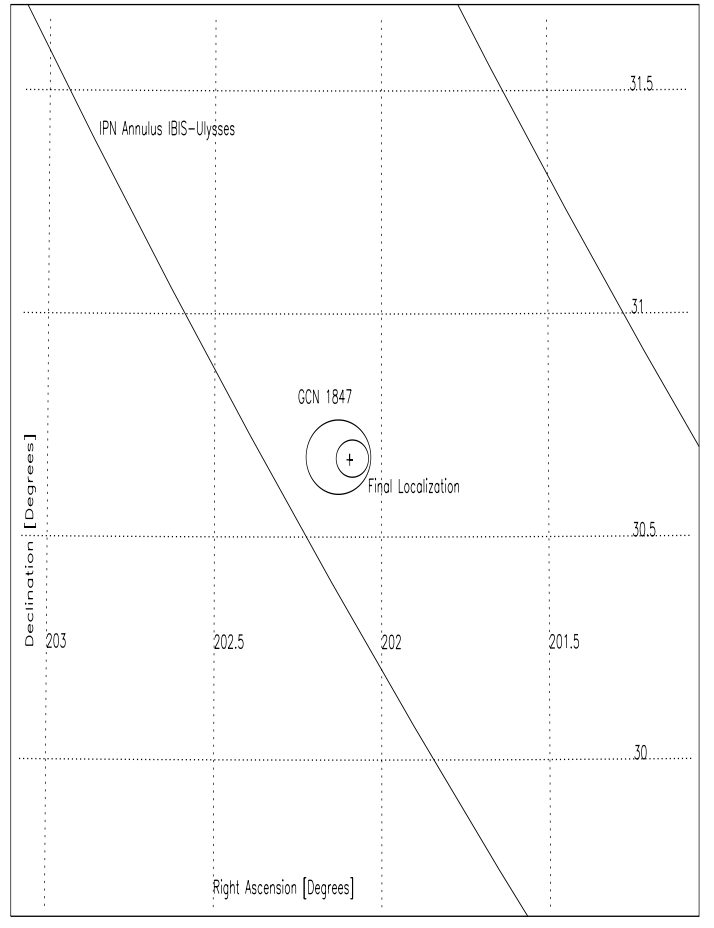

Fig. 1. Localizations of GRB 030131: the annulus obtained by the IPN is consistent with the localization given in Mereghetti et al. (2003a) (GCN 1847) and the one derived in this paper (the annulus obtained using SPI ACS and Ulysses is consistent with the one plotted but has a larger width). The cross indicates the position of the OT.

Telescope (VLT) with the FORS1 instrument at a mean date of 13 February 09:11:54 UTC. The seeing was about $1^{\prime \prime}$. There was no detectable object at the location of the candidate optical counterpart, with a $5 \sigma$ upper limit of $V>26.4$.

The marginal detection $\sim 29$ hours after the burst, with $B=$ $25.4 \pm 0.3$ (Gorosabel et al. 2003, but see also Henden 2003), and our VLT upper limit, confirm that this object is the OT of GRB 030131. Thus GRB 030131 is the first GRB detected with INTEGRAL with an associated optical counterpart.

\section{Temporal and spectral analysis}

We have analyzed IBIS/ISGRI single events, for which arrival time, energy deposit and interaction pixel of the detector are known for each event.

Figure 2 shows the light curves of GRB 030131 binned at $1 \mathrm{~s}$ resolution in different energy bands. The burst started at 07:38:49 UTC and lasted for about $150 \mathrm{~s}$. The time profile shows several peaks (note that the small gaps are artifacts caused by satellite telemetry saturation). The $T_{90}$ duration of the GRB in the $15-500 \mathrm{keV}$ band is $124 \mathrm{~s}$.

Since the GRB peaks during the satellite slew, we could not use the instrumental coordinates to extract the peak spectrum. Therefore we made an image selecting a time interval of $1 \mathrm{~s}$ around the GRB main peak $(t=54 \mathrm{~s}$ in Fig. 2). The high count rate at the peak allowed us to firmly establish the detector coordinates of the GRB even with this short integration time and thus to extract its peak spectrum. Since IBIS/ISGRI is a coded mask imaging instrument, the background can be estimated simultaneously with the source flux, using the Pixel Illumination Function (PIF; Skinner 1995). The spectra have 


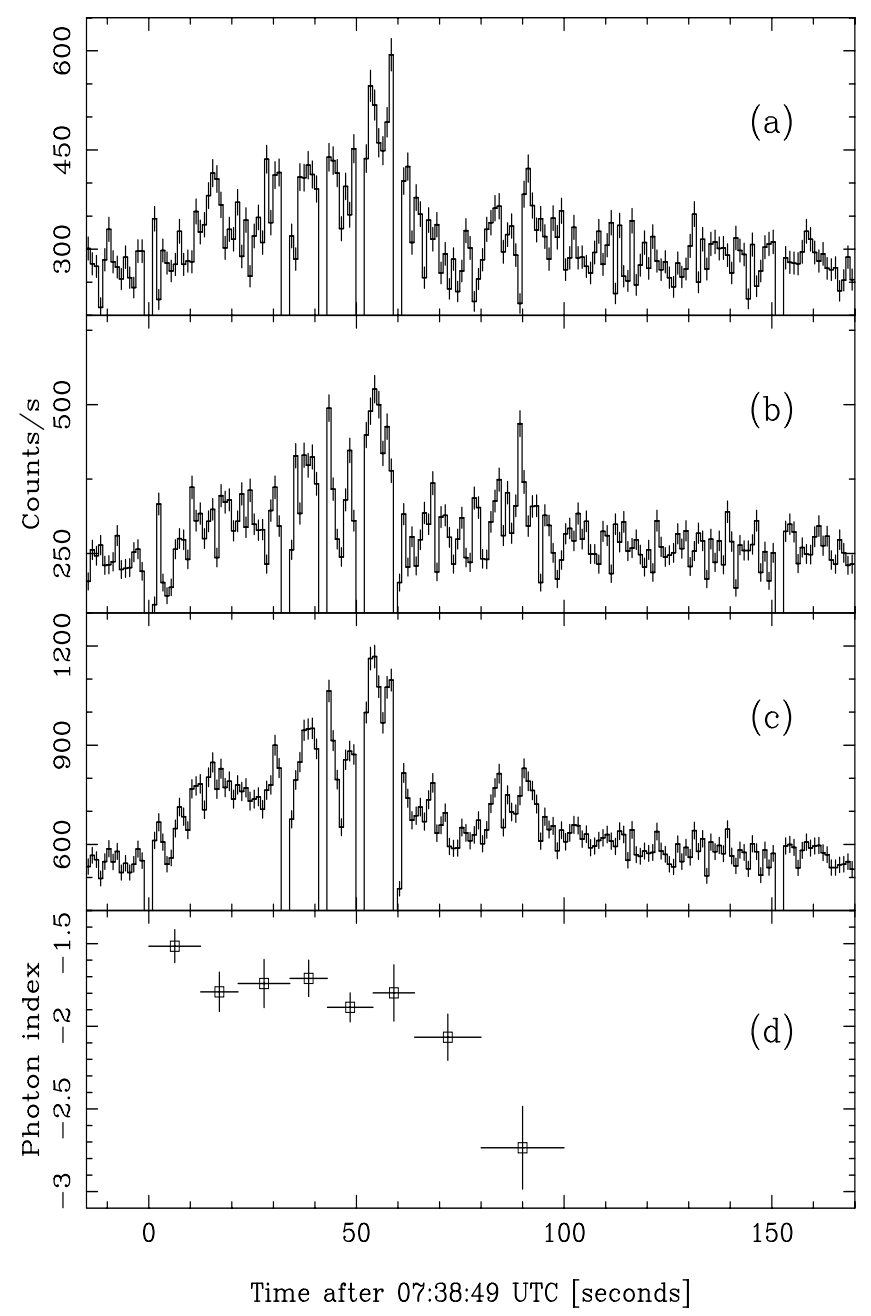

Fig. 2. IBIS/ISGRI light curve of GRB 030131 in various energy bands (a): $15-50 \mathrm{keV}, \mathbf{b})$ : $50-300 \mathrm{keV}, \mathbf{c}): 15-500 \mathrm{keV}$ ). The six data gaps are artifacts caused by satellite telemetry saturation. Four peaks can be identified, after $\sim 15, \sim 40, \sim 55, \sim 85 \mathrm{~s}$. The light curve has been corrected to take into account the varying fraction of the exposed instrument area during the satellite slew. Panel d) shows the spectral variation of the GRB with time.

been extracted computing one PIF for each energy bin (128 linearly spaced bins have been used between $19 \mathrm{keV}$ and $1 \mathrm{MeV}$ ). Since a fully calibrated spectral response matrix for sources at large off-axis angles is not yet available, we divided the count spectrum by the closest (in detector coordinates) count spectrum of the Crab Nebula. The resulting photon spectrum can be well fitted by a power law model $\left(\chi^{2} /\right.$ d.o.f. $\left.=8.66 / 8\right)$ with photon index $\Gamma=1.73_{-0.17}^{+0.16}$ (90\% confidence level). The flux is $\sim 1.9$ photons $\left(\sim 1.7 \times 10^{-7} \mathrm{erg}\right) \mathrm{cm}^{-2} \mathrm{~s}^{-1}(\sim 6.5 \mathrm{crab})$ in the $20-200 \mathrm{keV}$ energy range.

To properly extract the total GRB flux and spectrum, we derived the GRB detector coordinates at various time intervals for the entire duration of the event. The first interval, corresponding to the stable pointing, lasts $20 \mathrm{~s}$. The following 30 intervals last $3 \mathrm{~s}$ each and, finally, for the faint tail of the burst, four intervals with durations of $5,5,10$ and $20 \mathrm{~s}$ were used. The coordinates as a function of time are shown in Fig. 3, where

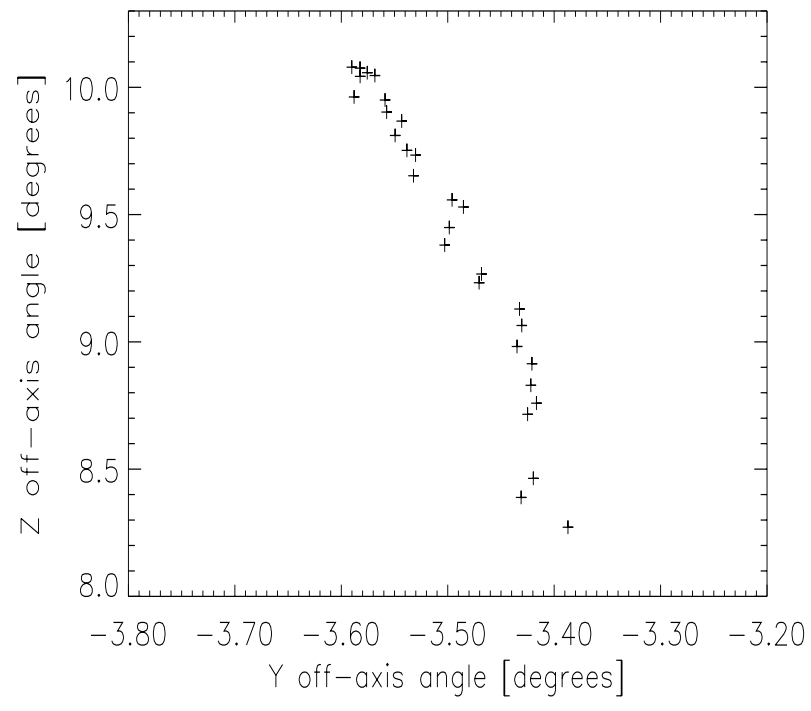

Fig. 3. Positions of GRB 030131 in detector coordinates as a function of time. Note the different scales of the two axes.

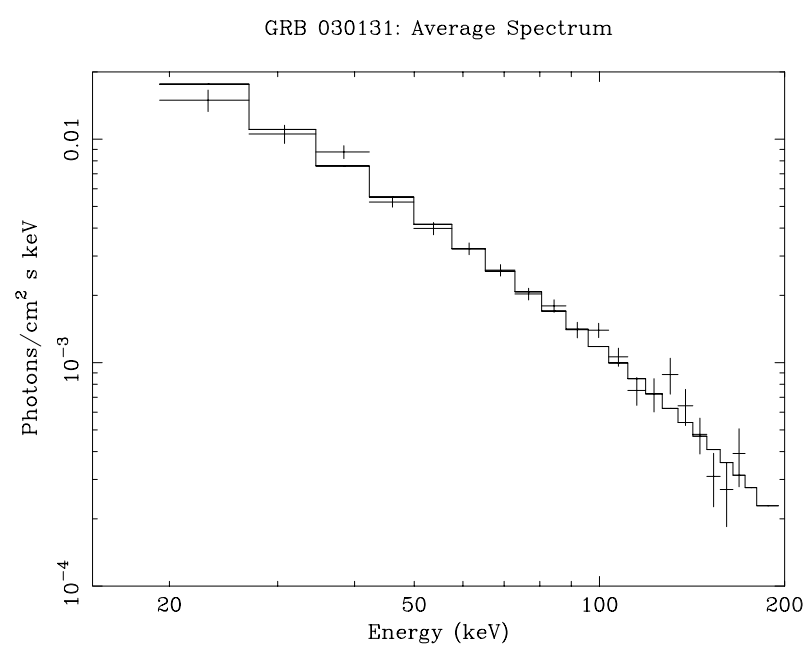

Fig. 4. Time averaged IBIS/ISGRI spectrum of GRB 030131. Data and best fit model are shown.

one can see the apparent drift of $\sim 2^{\circ}$ of the GRB in the IBIS field of view during the slew. To obtain the total (time averaged) spectrum, the 35 spectra extracted for the individual time intervals, corresponding to a net integration time of $147.2 \mathrm{~s}$, were co-added. We derived the photon spectrum (shown in Fig. 4) in the same way that we did for the peak spectrum. The GRB is clearly detected up to $200 \mathrm{keV}$. In this case a single power law model does not provide a satisfactory fit $\left(\chi^{2} /\right.$ d.o.f. $\left.=39.84 / 18\right)$, indicating that a model which includes a spectral break would give a better representation of the data. A fit using the Band model (Band et al. 1993) yields a break energy $E_{0}=70 \pm 20 \mathrm{keV}$, a low-energy power law index $\alpha=1.4 \pm 0.2$ and a high-energy photon index $\beta=3.0 \pm 1.0$ $\left(\chi^{2} /\right.$ d.o.f. $\left.=22.54 / 16\right)$. The fluence in the $20-200 \mathrm{keV}$ band is $\sim 73.8$ photons $\mathrm{cm}^{-2}\left(7 \times 10^{-6} \mathrm{erg} \mathrm{cm}^{-2}\right)$. To derive this value we have assumed that during the 4 central telemetry gaps the GRB had its average spectrum and intensity. Extrapolating the spectrum to the BATSE energy range (50-300 keV) we obtain a fluence of $\sim 23$ photons $\mathrm{cm}^{-2}\left(3.2 \times 10^{-6} \mathrm{erg} \mathrm{cm}^{-2}\right)$. The values 
of peak flux and fluence derived above are consistent within a factor of 2 with the ones measured with Ulysses. This indicates that our method does not suffer from large systematic errors and that the short telemetry gaps do not influence these values significantly.

We have also investigated the spectral evolution. As in the case of the peak spectrum, the data for the individual time intervals can be fitted with a single power law, without evidence for a spectral break. The photon index as a function of time is plotted in Fig. 2. A clear hard-to-soft evolution is seen. A hardening trend can also be seen corresponding to the rise of the second and third (main) peak with a softer spectrum for the latter peak. This correlation between light curve peaks and spectral hardening has already been reported in other bursts (e.g. Ford et al. 1995).

\section{Discussion}

The IBIS/ISGRI time-resolved spectroscopy of GRB 030131 is consistent with the overall hard-to-soft evolution observed with BATSE in many brighter GRBs, for which this kind of analysis was possible (e.g. Preece et al. 1998; Ford et al. 1995). The fluence of GRB 030131 is an order of magnitude smaller than those of the bursts studied by those authors, indicating that such spectral behaviour applies also to fainter GRBs. Clear evidence of this was also reported in the GRBs studied with BeppoSAX in the 2-700 keV energy range (e.g. Frontera et al. 2000; Frontera et al. 2003). While BATSE could better constrain the break energy and the high-energy slope of the Band function, thanks to its higher relative sensitivity above $200 \mathrm{keV}$, IBIS/ISGRI allows more detailed studies of the lowenergy part of the spectrum for relatively low fluence GRBs.

In the framework of the internal fireball shock model (Rees \& Mészáros 1994), and in particular of the Synchrotron Shock Model (Tavani 1996), the hard-to-soft evolution can be interpreted in two ways. The first possibility is a decrease of the magnetic field in the postshock region as a consequence of the postshock flow expansion; the second is a postshock decrease of the index of the particle distribution function as a consequence of strong cooling processes affecting the particle energy distribution for dynamical flow times larger than the radiating timescale. The two effects are not distinguishable in our case, since the time-resolved spectra do not have enough statistics to constrain a spectral break and hence a low-energy and a highenergy spectral index. The soft-to-hard evolution observed during the rise of the individual peaks, on the other hand, can be caused by an increase of the local magnetic field at the shock. Several authors reported that the duration of single pulses in GRB time histories is energy dependent (e.g. Link et al. 1993), with longer durations at lower energies, resulting in a hardening of the spectra before the peaks and a softening afterwards.

The optical transient associated with GRB 030131 indicates that we can classify it as an "optically dim" GRB. In fact it is as faint as (or even fainter than) the transient associated with GRB 030227, which is the only other INTEGRAL GRB with a firmly established optical conterpart, and was detected at $R \sim 23.312$ hours after the burst (Mereghetti et al. 2003b).
It is also comparable to GRB 021211. This event is also considered an optically dim burst since it was detected at $R \sim 18.2$ 1.3 hours after the prompt emission (Fox et al. 2003b) and was fainter than $R \sim 22.5$ after 12 hours (Klose et al. 2002). In addition GRB 030131 is located at much higher Galactic latitude $\left(b \simeq 81^{\circ}\right)$ which implies smaller foreground optical extinction. This indicates that, despite the efforts of observers, in some cases, optical follow-up with small telescopes is not an easy task, even less than 1 day after the burst (e.g Fynbo et al. 2001). The prompt localization of GRBs is hence a high priority in order to achieve a successful follow-up. The results on GRB 030501 (Beckmann et al. 2003) (an alert $30 \mathrm{~s}$ after the start of the GRB with an uncertainty of $4.4^{\prime}$; IBAS Alert 596) show that IBAS is now able to provide this service.

Acknowledgements. This research has been supported by the Italian Space Agency. KH is grateful for Ulysses support under JPL contract 958056, and for support of the IPN under NASA grant NAG5-12614. DHH acknowledges support by NASA. RH acknowledges the support by Prodex Project 14527 .

\section{References}

Band, D. L., Matteson, J., Ford, L., et al. 1993, ApJ, 413, 281

Beckmann, V., Borkowski, J., Courvoisier, T. J.-L., et al. 2003, A\&A, in press

Borkowski, J., Gotz, D., Mereghetti, S. Deluit, S., \& Walter, R. 2003, GCN 1836

Costa, E., Frontera, F., Heise, J., et al. 1997, Nature, 387, 783

Courvoisier, T., et al. 1999, in Proc. of the 3rd INTEGRAL Workshop - Astro Lett. \& Communications, 39, 355

Ford, L. A., Band, D. L., Matteson, J. L., et al. 1995, ApJ, 439, 307

Fox, D. W., Price, P. A., Herter, T., Appleton, P., \& Cotter, G. 2003a, GCN 1857

Fox, D. W., Price, P. A., Soderberg, A. M., et al. 2003b, ApJ, 586, L5

Frail, D., Kulkarni, S. R., Nicastro, S. R., Feroci, M., \& Taylor, G. B. 1997, Nature, 389, 261

Frontera, F., Amati, L., Costa, E., et al. 2000, ApJS, 127, 59

Frontera, F. et al. 2003, in preparation

Fynbo, J. U., Jensen, B. L., Gorosabel, J., et al. 2001, A\&A, 369, 373

Gorosabel, J., Levan. A., Fruchter, A., et al. 2003, GCN 1866

Henden, A. 2003, GCN 1971

Klebesadel, R. W., Strong, I. B., \& Olson, R. A. 1973, ApJ, 182, L85

Klose, S., Eisloeffel, J., Froebrich, D., et al. 2002, GCN 1739

Link, B., Esptein, R. I., \& Priedhorski, W. C. 1993, ApJ, 408, L81

Mereghetti, S., et al. 2001, in Proc. of the 4th INTEGRAL Workshop, ESA-SP, 459, 513

Mereghetti, S., Produit, N., Borkowski, J., \& Gotz, D. 2003a, GCN 1847

Mereghetti, S., Götz, D., Tiengo, A., et al. 2003b, ApJ, 590, L73

Preece, R. D., Pendleton, G. N., Briggs, M. S., et al. 1998, ApJ, 496, 849

Rees, M. J., \& Mészáros, P. 1994, ApJ, 430, L93

Ricker, G., Hurley, K., Lamb, D., et al. 2002, ApJ, 571, L127

Skinner, G. K. 1995, Experimental Atronomy, 6/4, 1

Tavani, M. 1996, ApJ, 466, 768

Ubertini, P., Lebrun, F., Di Cocco, G., et al. 1999, in Proc. of the 3rd INTEGRAL Workshop - Astro Lett. \& Communications, 39, 331 van Paradijs, J., Groot, P. J., Galama, T., et al. 1997, Nature, 386, 686 Vedrenne, G., Schönfelder, V., Albernhe, F., et al. 1999, in Proc. of the 3rd INTEGRAL Workshop - Astro Lett. \& Communications, 39, 325

Winkler, C. 1999, in Proc. of the 3rd INTEGRAL Workshop - Astro Lett. \& Communications, 39, 309 